\title{
Effect of process parameters on the surface roughness of aluminum alloy AA 6061-T6 sheets in frictional stir incremental forming
}

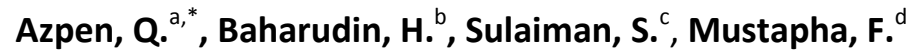 \\ $\mathrm{a}, \mathrm{b}, \mathrm{c}$ Universiti Putra Malaysia, Faculty of Engineering, Department of Mechanical and Manufacturing Engineering, Serdang, \\ Malaysia \\ dUniversiti Putra Malaysia, Faculty of Engineering, Department of Aerospace Engineering, Serdang, Malaysia \\ ${ }^{a}$ Middle Technical University, Institute of Technology, Baghdad, Iraq
}

\begin{abstract}
A B S TR ACT
Incremental Sheet Forming (ISF) is characterized by essential flexibility, great formability, and low forming forces and cost compared to the conventional sheet metal forming processes. ISF was born as an advance sheet metal forming process to perfectly fit previous requirements. Nevertheless, growing demand to apply the lightweight materials in several fields was placed this developed process in a critical challenge to manufacture the materials with unsatisfied formability especially at room temperature. Thus, utilizing the heat at warm and hot condition in some ISF processes has been introduced to solve this problem. Among all heat-assisted ISF processes, frictional stirassisted Single Point Incremental Forming (SPIF) was presented to deal with these materials. In this work, this emerging process was utilized to manufacturing products from AA6061-T6 aluminum alloy. Experimental tests were performed to study the influence of main parameters like tool rotation speed, feed rate, step size and tool size on the surface roughness of the produced parts. A Taguchi method and varying wall angle conical frustum (VWACF) test were used in the present work. The results find that tool diameter has a significant impact on the internal surface roughness produced via the forming process with a percentage contribution of $93.86 \%$. The minimum value of the surface roughness was $0.3 \mu \mathrm{m}$.
\end{abstract}

(C) 2018 CPE, University of Maribor. All rights reserved.

\author{
ARTICLE INFO \\ Keywords: \\ Friction stir forming; \\ Incremental sheet forming (ISF); \\ Heat-assisted ISF; \\ Surface roughness; \\ Aluminum alloy (AA6061-T6) \\ *Corresponding author: \\ qasimmhalhal@gmail.com \\ (Azpen, Q.) \\ Article history: \\ Received 11 July 2018 \\ Revised 26 August 2018 \\ Accepted 28 August 2018
}

\section{References}

[1] Ambrogio, G., Filice, L., Gagliardi, F. (2012). Formability of lightweight alloys by hot incremental sheet forming, Materials \& Design, Vol. 34, 501-508, doi: 10.1016/j.matdes.2011.08.024.

[2] Bao, W., Chu, X., Lin, S., Gao, J. (2015). Experimental investigation on formability and microstructure of AZ31B alloy in electropulse-assisted incremental forming, Materials \& Design, Vol. 87, 632-639, doi: 10.1016/i.matdes. 2015.08.072.

[3] Nee, A.Y.C. (2015). Handbook of manufacturing engineering and technology, Springer, London, UK, doi: 10.1007/ 978-1-4471-4670-4.

[4] Xu, D., Lu, B., Cao, T., Chen, J., Long, H., Cao, J. (2014). A comparative study on process potentials for frictional stir-and electric hot-assisted incremental sheet forming, Procedia Engineering, Vol. 81, 2324-2329, doi: 10.1016/i.proeng.2014.10.328.

[5] Asghar, J., Reddy, N.V. (2013). Importance of tool configuration in incremental sheet metal forming of difficult to form materials using electro-plasticity, In: World Congress on Engineering, WCE 2013, London, United Kingdom. 
[6] Durante, M., Formisano, A., Langella, A., Minutolo, F.M.C. (2009). The influence of tool rotation on an incremental forming process, Journal of Materials Processing Technology, Vol. 209, No. 9, 4621-4626, doi: 10.1016/i. jmatprotec.2008.11.028.

[7] Baharudin, B.T.H.T., Azpen, Q.M., Sulaima, S., Mustapha, F. (2017). Experimental investigation of forming forces in frictional stir incremental forming of aluminum alloy AA6061-T6, Metals, Vol. 7, No. 11, 484, doi: $10.3390 /$ met7110484.

[8] Bagudanch, I., Centeno, G., Vallellano, C., Garcia-Romeu, M.L. (2013). Forming force in single point incremental forming under different bending conditions, Procedia Engineering, Vol. 63, 354-360, doi: 10.1016/i.proeng. 2013.08.207.

[9] Hagan, E., Jeswiet, J. (2004). Analysis of surface roughness for parts formed by computer numerical controlled incremental forming, Proceedings of the Institution of Mechanical Engineers, Part B: Journal of Engineering Manufacture, Vol. 218, No. 10, 1307-1312, doi: 10.1243/0954405042323559.

[10] Jeswiet, J., Micari, F., Hirt, G., Bramley, A., Duflou, J., Allwood, J. (2005). Asymmetric single point incremental forming of sheet metal, CIRP Annals, Vol. 54, No. 2, 88-114, doi: 10.1016/S0007-8506(07)60021-3.

[11] Hamilton, K., Jeswiet, J. (2010). Single point incremental forming at high feed rates and rotational speeds: Surface and structural consequences, CIRP Annals, Vol. 59, No. 1, 311-314, doi: 10.1016/i.cirp.2010.03.016.

[12] Oleksik, V., Pascu, A., Deac, C., Fleacă, R., Bologa, O., Racz, G. (2010). Experimental study on the surface quality of the medical implants obtained by single point incremental forming, International Journal of Material Forming, Vol. 3, Supplement 1, 935-938, doi: 10.1007/s12289-010-0922-x.

[13] Bhattacharya, A., Maneesh, K., Venkata Reddy, N., Cao, J. (2011). Formability and surface finish studies in single point incremental forming, Journal of Manufacturing Science and Engineering, Vol. 133, No. 6, doi: 10.1115/ 1.4005458.

[14] Palumbo, G., Brandizzi, M. (2012). Experimental investigations on the single point incremental forming of a titanium alloy component combining static heating with high tool rotation speed, Materials \& Design, Vol. 40, 4351, doi: 10.1016/i.matdes.2012.03.031.

[15] Ambrogio, G., Filice, L., Gagliardi, F. (2012). Improving industrial suitability of incremental sheet forming process, The International Journal of Advanced Manufacturing Technology, Vol. 58, No. 9-12, 941-947, doi: 10.1007/ s00170-011-3448-6.

[16] Silva, P.J., Leodido, L.M., Silva, C.R.M. (2013). Analysis of incremental sheet forming parameters and tools aimed at rapid prototyping, Key Engineering Materials, Vol. 554-557, 2285-2292, doi: 10.4028/www.scientific.net/ KEM.554-557.2285.

[17] Lasunon, 0.U. (2013). Surface roughness in incremental sheet metal forming of AA5052, Advanced Materials Research, Vol. 753-755, 203-206, doi: 10.4028/www.scientific.net/AMR.753-755.203.

[18] Filice, L., Fratini, L., Micari, F. (2002). Analysis of material formability in incremental forming, CIRP Annals, Vol. 51, No. 1, 199-202, doi: 10.1016/S0007-8506(07)61499-1.

[19] Skjoedt, M., Hancock, M.H., Bay, N. (2007). Creating helical tool paths for single point incremental forming, Key Engineering Materials, Vol. 344, 583-590, doi: 10.4028/www.scientific.net/KEM.344.583.

[20] Lu, B., Chen, J., Ou, H., Cao, J. (2013). Feature-based tool path generation approach for incremental sheet forming process, Journal of Materials Processing Technology, Vol. 213, No. 7, 1221-1233, doi: 10.1016/i.jmatprotec. 2013.01.023.

[21] Liu, Z., Liu, S., Li, Y., Meehan, P.A. (2014). Modeling and optimization of surface roughness in incremental sheet forming using a multi-objective function, Materials and Manufacturing Processes, Vol. 29, No. 7, 808-818, doi: $10.1080 / 10426914.2013 .864405$.

[22] Mugendiran, V., Gnanavelbabu, A., Ramadoss, R. (2014). Parameter optimization for surface roughness and wall thickness on AA5052 aluminium alloy by incremental forming using response surface methodology, Procedia Engineering, Vol. 97, 1991-2000, doi: 10.1016/j.proeng.2014.12.442.

[23] Lu, B., Fang, Y., Xu, D.K., Chen, J., Ou, H., Moser, N.H., Cao, J. (2014). Mechanism investigation of friction-related effects in single point incremental forming using a developed oblique roller-ball tool, International Journal of Machine Tools and Manufacture, Vol. 85, 14-29, doi: 10.1016/j.ijmachtools.2014.04.007.

[24] Azevedo, N.G., Farias, J.S., Bastos, R.P., Teixeira, P., Davim, J.P., de Sousa, R.J.A. (2015). Lubrication aspects during single point incremental forming for steel and aluminum materials, International Journal of Precision Engineering and Manufacturing, Vol. 16, No. 3, 589-595, doi: 10.1007/s12541-015-0079-0.

[25] Reddy, N.V., Cao, J. (2008). Incremental sheet metal forming: A review, In: Proceedings of the Indo-US workshop on Smart Machine Tools, Intelligent Manufacturing Systems at Multiscale Manufacturing, PSG college of Technology, Combotore, India.

[26] Petek, A., Kuzman, K., Kopač, J. (2009). Deformations and forces analysis of single point incremental sheet metal forming, Archives of Materials Science and Engineering, Vol. 35, No. 2, 35-42.

[27] Buffa, G., Campanella, D., Fratini, L. (2013). On the improvement of material formability in SPIF operation through tool stirring action, The International Journal of Advanced Manufacturing Technology, Vol. 66, No. 9-12, 1343-1351, doi: $10.1007 / \mathrm{s} 00170-012-4412-9$. 


\title{
Vpliv procesnih parametrov na površinsko hrapavost pločevine iz aluminijeve zlitine AA 6061-T6 pri vodenem tornem inkrementalnem preoblikovanju
}

\author{
Azpen, Q..$^{a}{ }^{,}$, Baharudin, H. ${ }^{b}$, Sulaiman, S. $^{c}$, Mustapha, F. ${ }^{d}$ \\ a,b,c Universiti Putra Malaysia, Faculty of Engineering, Department of Mechanical and Manufacturing Engineering, Serdang, \\ Malaysia \\ dUniversiti Putra Malaysia, Faculty of Engineering, Department of Aerospace Engineering, Serdang, Malaysia \\ ${ }^{a}$ Middle Technical University, Institute of Technology, Baghdad, Iraq
}

\begin{abstract}
POVZETEK
Za inkrementalno preoblikovanje pločevine (ISF) je značilna prilagodljivost, velika sposobnost preoblikovanja ter nizke preoblikovalne sile in stroški v primerjavi s konvencionalnimi postopki preoblikovanja pločevine. ISF se je pojavil kot napredni postopek preoblikovanja pločevine $\mathrm{z}$ namenom popolnoma zadostiti obstoječim zahtevam. Naraščajoče povpraševanje po lahkih materialih je postavilo razvoj procesa pred kritičen izziv, saj se je na mnogih področjih pojavila zahteva po izdelkih iz materialov z nezadovoljivo sposobnostjo preoblikovanja, zlasti pri sobni temperaturi. Zato je bila za reševanje tega problema pri nekaterih procesih ISF vpeljana uporaba toplote za preoblikovanje pri toplih in vročih pogojih. Za obravnavo takšnih materialov je bil, med vsemi s toploto podprtimi procesi ISF, izbran proces vodenega točkovnega tornega inkrementalnega preoblikovanja (SPIF). V članku je predstavljena uporaba tega nastajajočega procesa za proizvodnjo izdelkov iz aluminijeve zlitine AA6061-T6. Izvedeni so bili eksperimentalni testi za preučevanje vpliva glavnih parametrov, kot so vrtilna hitrost orodja, hitrost podajanja, velikost korakov in velikost orodja na površinsko hrapavost izdelkov. V članku smo opisali teste izvedene po Taguchi metodi, na odrezanem stožcu s spremenljivo koničnostjo. Rezultati kažejo, da ima premer orodja pomemben vpliv na hrapavost notranje površine, ki nastane s postopkom preoblikovanja, s prispevkom 93,86 \%. Najmanjša vrednost površinske hrapavosti je bila 0,3 $\mu \mathrm{m}$
\end{abstract}

\section{PODATKI O ČLANKU}

\section{Ključne besede:}

Vodeno torno preoblikovanje;

Inkrementalno preoblikovanje

Toplotno podprto inkrementalno preoblikovanje;

Površinska hrapavost;

Aluminijeva zlitina (AA6061-T6)

*Kontaktna oseba: qasimmhalhal@gmail.com (Azpen, Q.)

Zgodovina članka: Prejet 11. julija 2018

Popravljen 26. avgusta 2018 Sprejet 28. avgusta 2018 